\title{
POBLACIÓN Y AMBIENTE: CUESTIONES ACTUALES
}

Susana B. Adamo*

Aula proferida pela Profa. Dra. Susana B. Adamo no dia 26 de março de 2014 no Núcleo de Estudos de População “Elza Berquó" (NEPO/UNICAMP). ${ }^{* *}$

\section{El campo de estudio}

El campo de estudio de población y ambiente trabaja esencialmente con las interacciones de los procesos demográficos y ambientales, incluyendo sus múltiples dimensiones y determinaciones. Se caracteriza por una gran variedad de temas de investigación, abarcando problemáticas tan diversas como crecimiento y distribución de la población, estilos de desarrollo, exposición a la contaminación, cambios en el uso del suelo, salud y epidemiología, y últimamente ha incorporado también cuestiones de seguridad, conflictos y desplazamientos internos de la población. Debido a esta diversidad, este campo toma prestados elementos teóricos y metodológicos de un número de disciplinas científicas, sociales y naturales, entre ellas demografía, sociología,

\footnotetext{
* Center for International Earth Science Information Network (CIESIN) Columbia University, Estados Unidos. (sadamo@ciesin.columbia.edu).

** Os editores da Idéias - Revista do Instituto de Filosofia e Ciências Humanas da UNICAMP - agradecem a disponibilidade da professora Susana Adamo em transformar sua aula em texto e ceder a esta Revista a oportunidade de publicá-lo.
} 
ecología, ciencias ambientales, economía, geografía, climatología, biología, e historia

“[... El campo de las interacciones
entre población y ambiente [...] es
complejo y diverso, con capacidad de
estudio a diferentes niveles, desde lo
local a lo global, y desde diferentes
puntos de vista: el demográfico, el social
y económico, el geográfico, el ecológico
y ambiental [...]" (ZABA; CLAR-
KE, 1994: 36, traducción propia).

A pesar de sus límites borrosos, Lutz y otros (2002: 2) afirman que esta área de conocimiento constituye un 'campo de investigación científica' ya que cumple con dos de tres requisitos mínimos necesarios. En primer lugar, reconoce una masa crítica de investigadores trabajando en el área. En segundo lugar, tiene preguntas de investigación comunes, centradas en dos ejes: cuál es el impacto de las estructuras y los cambios poblacionales sobre el medio ambiente, y cuál es el impacto de los cambios ambientales sobre la población y sus procesos demográficos. Según los autores, el campo aún debe cumplir con la tercera condición, que es la de tener una metodología común. Aunque ha habido avances importantes en este sentido en los últimos años (véase por ejemplo FUSSELL; HUNTER; GRAY, 2014; HUMMEL et al., 2013), la discusión teórico-metodológica es una preocupación constante en este campo de trabajo.

La multiplicidad disciplinaria, teórica y metodológica implica que las problemáticas incluidas en este campo de estudio no son fáciles de aprehender o definir, e incluso han sido declaradas algunas veces como "inmanejables" desde el punto de vista de la investigación. Las cuestiones de población y ambiente son sumamente complejas ya que las mismas involucran la consideración de varios y diversos componentes, los cuales son objeto de diferentes disciplinas sociales y naturales, hecho 
que plantea desafíos particulares desde el punto de vista de la interdisciplinariedad. Estos elementos están interconectados, lo cual conlleva que la consideración de su interdependencia y articulación son aspectos esenciales en la definición del objeto de estudio. Por último, se tiene la insoslayable presencia de las dimensiones temporal y espacial, lo que obliga a considerar los conceptos de proceso, contexto, escala y jerarquía (ADAMO 2007; HUMMEL et al., 2013).

\section{Cómo se articulan o relacionan las dinámicas demográfica y} ambiental?

Las dos aproximaciones conceptuales clásicas a la relación población-medio ambiente están representadas por Thomas Malthus y Ester Boserup. Malthus (1826) formuló una teoría de población y ambiente centrado en la habilidad del ambiente de proveer o producir alimentos, la cual consideró aplicable a cualquier tipo o forma de población humana sin distinciones de escala. En este marco conceptual, la población crecería en forma geométrica, mientras que la productividad de la agricultura se incrementaría linealmente a lo largo del tiempo. La clave del bienestar estaría en la razón entre la población y los rendimientos de la agricultura. Si la razón aumenta, el bienestar declina. Frente a esto, existirían controles (checks) preventivos - usos y costumbres que regulan la fecundidad tales como edad al matrimonio, proporción de mujeres que entran en unión, infanticidio, emigración, etc. - y positivos - hambre, enfermedades, conflictos sobre la tierra, etc. (DE SHERBININ et al., 2007). VanWey et al. (2005) sostienen que la teoría de Malthus adolece de dos problemas que se trasladan a aproximaciones conceptuales basadas en la misma como el neomalthusianismo (que sostiene que la población es la causa principal de cambios ambientales negativos, véase por ejemplo Marandola; Hogan 2007, de Sherbinin et al., 2007). En primer lugar, no permite la posibilidad del crecimiento no linear de la productividad de la agricultura, por ejemplo en respuesta a nuevas 
tecnologías. En segundo lugar, y en relación directa con lo anterior, no incluye la innovación agrícola como una posible respuesta a la escasez, sino que postula que la misma llevaría directamente a la disminución de la población.

Boserup ([1965] 2005) desafío la idea que población o tecnología eran la fuerza dominante detrás de cambios en la agricultura y desarrollo, y en cambio postuló que son los incrementos en la densidad de población que llevan a la escasez relativa de tierras los que actúan como disparadores o estímulo para la intensificación de la agricultura (o sea, el incremento de la productividad en tierras ya bajo cultivo a través de, por ejemplo, la implementación de sistemas de riego o la reducción del tiempo bajo barbecho), centrándose en las consecuencias y no en las causas del cambio poblacional. La autora argumenta que los productores que no enfrentan situaciones de escasez no están motivados para buscar formas de incrementar la productividad, mientras que al enfrentarse a una densidad poblacional creciente incrementarían el esfuerzo (terrazas, irrigación, fertilizantes, etc.). A partir de estos conceptos, no habría techo agrario o capacidad de soporte natural (carrying capacity) para un ambiente o región en particular. El nivel de productividad que se puede alcanzar en un lugar dado depende de la tecnología (capital físico) pero también del capital social y humano. Sin embargo, es necesario tomar en cuenta la complejidad del proceso de innovación, ya que cambiar o modificar tecnologías en ciertos ambientes requiere considerable experimentación e inversiones en nuevas herramientas y técnicas. Algunos grupos se pueden ver enfrentados con cambios tan repentinos en población y/o en la disponibilidad de recursos que podrían no ajustarse lo suficientemente rápido, lo cual podría, paradójicamente, llevar a situaciones cercanas a las indicadas por Malthus (DE SHERBININ et al., 2007: 4). Los seguidores de Boserup se han focalizado en entender como la escasez de recursos podría estimular cambios tecnológicos que a sus vez lleven a cambios positivos y no negativos en el ambiente (VANWEY et al., 2005).

A partir de las concepciones clásicas, lo largo del tiempo se han desarrollado distintas aproximaciones teóricas para modelar 
la relación población-ambiente. Basado en Marquette (1997) y Hummel et al. (2013), las mismas se pueden resumir en las siguientes categorías:

- Aproximaciones lineales: las mismas enfatizan relaciones lineales recíprocas, directas, causales y determinísticas entre población y ambiente. Entreellas se incluyen posturas malthusianas, neo-malthusianas, pero también aquellas basadas en el trabajo de Boserup.

- Aproximaciones multiplicativas: el postulado básico es que la población (representada solo por su tamaño) interactúa en forma multiplicativa con otros factores para afectar o impactar el ambiente. De este modo, la población es un elemento central pero ligado a la actividad económica y las técnicas. Una de las aproximaciones más usadas es la ecuación "IPAT": los impactos sobre el ambiente (I) son el resultado de la población (P) multiplicada por el nivel de consumo per cápita o afluencia (A) y por el nivel tecnológico (T). Desarrollos más recientes del modelo IPAT incluyen STIRPAT (Stochastic Impacts by Regression on Population, Affluence, and Technology), el cual mantiene los fundamentos ecológicos y la lógica multiplicativa de IPAT, pero agrega (1) la estimación del efecto neto de las fuerzas antropogénicas sobre el ambiente, (2) el testeo de hipótesis y modelos, y (3) la incorporación de otras variables teóricamente relevantes como factores políticos, sociales y culturales.

- Aproximaciones mediadas: el énfasis es en los contextos (incluyendo los factores sociales, culturales, institucionales, políticos, económico, científicos, etc.) en el cual se da la relación entre población y ambiente. Se postula que no hay una relación directa entre población y ambiente, sino que estos factores mediadores (a su vez interrelacionados) conectan o articulan estructuras y dinámicas poblacionales con sus contrapartes ambientales. Una de las perspectivas incluidas es la ecología política, la cual se refiere a una colección de perspectivas que buscan hacer explícita la interacción entre procesos políticos y ecológicos actuando a diferentes escalas geográficas y temporales. Otra perspectiva incluida es la de medios de vida sustentables, la 
cual considera al hogar (dentro de la comunidad) como la unidad de análisis fundamental, y examina cómo el acceso a diferentes activos o recursos puede ser convertido en estrategias de vida, dentro de contextos de vulnerabilidad y marcos institucionales específicos.

- Aproximaciones basadas en sistemas complejos: postulan que las relaciones entre población y ambiente se estructuran y a su vez están embebidas dentro de un complejo de sistemas interrelacionados. El objetivo es entender cómo una diversidad de sistemas ecológicos y humanos (socioculturales, demográficos, económicos) se interconectan y articulan para formar grandes sistemas socio-ecológicos. Desde la teoría de sistemas, estas perspectivas toman en cuenta dinámicas, agentes que se adaptan a situaciones cambiantes, y procesos que co-evolucionan. Este giro hacia la consideración de sistemas más complejos es influenciado por investigación en ecología y ambiente pero también en desarrollo y reducción de la pobreza. Las diversas perspectivas incluidas en estas aproximaciones tienen en común el considerar la población y el ambiente como sistemas interactivos, y el centrarse en la interdependencia de cambios ambientales y sociales. Ejemplos de estas perspectivas incluyen CHANS (coupled human and natural systems), PEDA (population-environment-development model), "supply systems", ecología de la población, ecología humana, y ecología cultural, entre otras (HUMMEL et al., 2013).

En términos de las estrategias metodológicas utilizadas, comparten la misma diversidad, yendo desde la regresión lineal hasta los estudios de caso participativos, de las críticas etnográficas (ecología política) a las simulaciones, y de los estudios de casos históricos a los escenarios de sistemas interactivos humanos/ ecológicos (por ejemplo, CHANS y PEDA). Algunos enfoques (por ejemplo, la ecología política o supply systems) son útiles para explicar procesos en un lugar específico, pero es difícil generalizar los resultados a otros contextos. Otros enfoques, en cambio, permiten generalizaciones y proyecciones a partir del análisis de una política específica (por ejemplo, STIRPAT), pero están más alejados de las experiencias vividas. 
En resumen, no existen "panaceas" teóricas o metodológicas en el estudio de las interacciones población-ambiente. Diferentes enfoques conceptuales no son inevitablemente mutuamente excluyentes, sino que se pueden combinar y complementar entre sí. La selección de una perspectiva determinada va a depender de: el objetivo de estudio de los investigadores; cuanta atención deba prestarse a cuestiones de escala, interacciones, y una amplia gama de dinámicas sociales, demográficas y ecológicas; y la necesidad de comunicar patrones de sistemas complejos a responsables políticos o agentes sociales interesados buscando puntos simples e identificables para intervenciones. En todos los casos, es muy importante explorar la combinación de enfoques y metodologías (por ejemplo métodos cualitativos y cuantitativos, o la integración de fuentes tradicionales con información geo-referenciada).

\section{Cuestiones actuales}

Se pueden mencionar tres cuestiones que actualmente atraen la atención de los investigadores: (1) la dinámica poblacional en el marco del desarrollo sustentable; (2) el rol de la dinámica demográfica en procesos de vulnerabilidad, mitigación, y adaptación en el contexto del cambio ambiental global, incluyendo cambio climático; y (3) dinámica demográfica y medio ambiente, incluyendo migración y desplazamientos ambientales, crecimiento demográfico, consumo, y re-distribución.

\section{(1) Población, ambiente y desarrollo sustentable}

La preocupación por la falta de mención explícita de los temas de población dentro de los temas principales de la Conferencia de la Naciones Unidas sobre Desarrollo Sustentable Rio+20, llevó a un grupo de demógrafos a elaborar un documento, la Declaración de Laxenburg (GLOBAL EXPERT PANEL, 2011) solicitando y justificando la inclusión de la dinámica demográfica -tamaño, características, estructura, composición, distribución, 


\section{En que estamos trabajando?}

A modo de ejemplo, estos son las sesiones sobre población y ambiente en reuniones científicas recientes:

- XXVII Conferencia Internacional de Población IUSSP 2013: dinámica de la población $\mathrm{y}$ cambio climático; población y escenarios socioeconómicos para la investigación del cambio climático; dinámica poblacional y linkages ambientales; amenazas ambientales a la salud infantil; impacto de cambios ambientales y climáticos sobre la salud y la mortalidad; fecundidad y salud reproductiva: examinando links con el ambiente; mapeo del riesgo; población y medio ambiente; la dinámica población-ambiente en América Latina; migración como forma de adaptación a eventos climáticos extremos, desastres naturales y cambio ambiental (http://goo.gl/aszVYx).

- VI Congreso ALAP 2014: poblacion y desarrollo sostenible: ciudades, dinámica de la población y conexión con medio ambiente; dinámica de población y cambio climático; demographic dynamics, environmental change and health in the Amazon; población y ambiente; la relación entre salud, medio ambiente y demografía; políticas públicas urbanas $e$ mudanças climáticas na América Latina: vulnerabilidade, adaptação $e$ resiliência (http://goo.gl/0cQSg6). procesos de cambio - en la agenda de la Conferencia ${ }^{1}$. El argumento principal es que todo análisis del desarrollo sustentable debe reconocer las diferencias que existen entre diversos grupos sociales en relación a su impacto sobre el ambiente y su vulnerabilidad al riesgo ambiental, lo cual depende de su edad, genero, localización y características socioeconómicas. Por ejemplo, nueva evidencia sugiere que la educación y la salud (incluyendo salud reproductiva) están relacionadas con la contribución de los individuos al desarrollo sustentable, y a su capacidad de adaptación al cambio ambiental. Similarmente, la transición a una sociedad cada vez más urbana podría contribuir a minimizar riesgos, peligros, vulnerabilidad y degradación ambiental.

En el marco de esta preocupación por integrar la dinámica demográfica en la discusión sobre desarrollo sustentable, un libro reciente sobrepoblacióny sustentabilidad en Brasil (MARTINE, 2012)

\footnotetext{
${ }^{1}$ En el último año, este tema se actualizó en el marco de las metas de desarrollo sustentable y durante la Sesión $48^{\underline{a}}$ de la Comisión de Población y Desarrollo de Naciones Unidas (http://goo.gl/UK2qta).
} 
sostiene que las características, estructuras y cambios demográficos deben estar presentes en el debate ambiental porque influyen decisivamente en las actividades productivas y en los patrones de consumo. Esto sería particularmente relevante en Brasil, cuya población ha experimentado una muy rápida transición de la fecundidad, y una aun más rápida transición urbana, así como también la reducción de la pobreza y la desigualdad. El contenido del libro avanza un paso más, al proponerse también 'traducir' los resultados de la investigación científica a información que pueda realmente usarse para la acción, evitando la jerga académica para llegar a una audiencia más amplia, "tendiendo puentes" entre el conocimiento científico y el público, y articulando las escalas global y nacional, y las cuestiones demográficas y ambientales. Su objetivo final es la difusión del conocimiento científico de las relaciones entre dinámica demográfica, procesos de desarrollo y medio ambiente. Los temas tratados y las perspectivas elegidas son muy diversos, pero se puede detectar un hilo conductor: la interacción de la dinámica demográfica con los procesos de desarrollo, la cual a su vez influye en los cambios ambientales.

\section{(2) Cambio climático, vulnerabilidad, adaptación y resiliencia}

El tema de la vulnerabilidad de grupos poblacionales a peligros ambientales (hazards) ha formado parte de la agenda de este campo de estudios desde hace tiempo (véase por ejemplo Blaikie et al., 1996), pero se ha revitalizado en el marco del cambio climático. Marandola y Hogan (2009:163) sostienen que es fundamental preguntarse "vulnerabilidad a que?" ya que de esta forma se llega a los "grupos demográficos que estão sujeitos a determinados perigos, que podem estar relacionados às características da dinâmica demográfica ou à sua situação socioeconômica, ligadas ao ciclo vital, à estrutura familiar ou às características migratórias do grupo". Los autores consideran que es igualmente importante tomar en cuenta la dimensión espacial de la problemática población-ambiente, considerando “a posição e a situação (relacionais e relativas) componentes dos elementos 
que produzem perigos ou que fornecem condições de enfrentálos" (2009: 164). Proponen estrategias de investigación basadas en la vulnerabilidad de los lugares, la cual permitiría, en una escala espacial micro, "captar os elementos que interferem na produção, aceitação e mitigação dos riscos. A dimensão ecológica é re-significada ao incorporar a dimensão existencial e fenomênica do lugar, entendendo os grupos demográficos em sua relação de envolvimento e pertencimento ao seu espaço vivido" (2009: 165)

En relación a cambios ambientales, el World Social Science Report 2013 afirma que "global environmental change is about humans changing global environments, and about humans, individually and collectively, shaping the direction of planetary and social evolution" (ISSC/UNESCO, 2013: 4). Esta concepción está detrás del creciente interés en los temas de adaptación dentro de las ciencias sociales y especialmente en relación al desarrollo sustentable (como se comento en la sección anterior). Sin embargo, como afirman Schensul y Dodman (2013) se necesita también incorporar la dinámica poblacional para evitar crear una brecha entre el desarrollo y la implementación de proyectos de adaptación. En efecto, la dinámica demográfica es crítica para entender la distribución y diferenciación de la vulnerabilidad, la exposición y la capacidad de adaptación entre diferentes grupos sociales. Igualmente, se debería incorporar la dinámica poblacional en políticas y planeación relacionados con estos procesos y con las estrategias para la reducción de desastres y prevención del riesgo. A modo de ejemplo, ciertos aspectos de la dinámica poblacional, como por ejemplo la distribución por edad o la distribución espacial, tienen una conexión directa a la adaptación (véase por ejemplo CEPAL, 2012), en tanto que las proyecciones de población pueden proveer escenarios plausibles sobre el tamaño y la composición de la población en el futuro, lo cual tiene importantes implicaciones para las políticas de mitigación, adaptación y prevención.

Más y más aspectos de la dinámica demográfica están siendo incorporados dentro de los modelos de cambio climático. Como afirman Hunter y O'Neill (2014), “demographers have much to contribute to climate change science". En su artículo, estos autores 
presentan un nuevo marco para estos modelos, desarrollado por la comunidad de climatólogos. Los shared socio-economic pathways (SSPs) (que se podría traducir libremente como vías socioeconómicas compartidas) representan "tendencias alternativas plausibles en la evolución de los sistemas sociales y naturales durante el siglo XXI a escala mundial y de grandes regiones." (HUNTER; O'NEILL, 2014: 232). Estos escenarios podrían convertirse en una herramienta valiosa para la organización de la investigación en el campo de población-ambiente al identificar la intersección de tendencias que podrían influir tendencias futuras en el clima, y que requieren más investigación, por ejemplo urbanización y consumo energético. También pueden ser utilizados para aumentar la relevancia de los estudios de caso locales para la elaboración de políticas, ofreciendo un nivel básico de armonización que puede facilitar la generalización, como una nueva forma de meta-análisis. De esta forma, el marco de los SSPs puede aumentar la relevancia de los resultados de la investigación demográfica para las políticas de mitigación y adaptación, y también hacerlos más accesibles a otras comunidades científicas.

\section{(3) Dinámica demográfica y medio ambiente}

Diversas características de la dinámica demográfica actual son relevantes para entender posibles cambios en la relación población-ambiente. Para empezar, el momento actual se caracteriza por tendencias globales y especificidades locales, como se ejemplifica en las diferencias regionales que se observan en la Tabla 1. En segundo lugar, tenemos los cambios en la distribución espacial de la población, como se observa en la Figura 1. Estos cambios están relacionados con una creciente movilidad espacial y con el proceso de transición urbana, o el paso de una población predominantemente rural a una predominantemente urbana. Esta transición está bastante avanzada en América Latina, como se observa en la Figura 2: desde la década del 1960, la población de la región es mayoritariamente urbana, si bien con importantes diferencias entre países. 
Población y ambiente: cuestiones actuales...

Estos cambios en dinámica y estructura están fuertemente relacionados con cambios en los patrones de consumo, incluidos cambios en dietas (por ejemplo mayor consumo de carne), aumento del consumo de bienes durables (como electrodomésticos y automóviles), y aceleración de ciclos de reemplazo (por ejemplo, en los casos de teléfonos celulares y otros bienes relacionados con las nuevas tecnologías de información y comunicación, que contribuyen a la proliferación dela"basura electrónica"(LEVY;MOREL, 2012:24).

TABELA 1 - Información demográfica, por regiones del mundo, 2011

\begin{tabular}{c|c|c|c|c|c|c|c}
\hline Indicador & África & Oceanía & Asia & Europa & $\begin{array}{c}\text { América } \\
\text { Latina y el } \\
\text { Caribe }\end{array}$ & $\begin{array}{c}\text { América } \\
\text { del Norte }\end{array}$ & $\begin{array}{c}\text { Global } \\
\text { (países con } \\
\text { información) }\end{array}$ \\
\hline $\begin{array}{c}\text { Tasa de natalidad } \\
\text { (por 1.000) }\end{array}$ & 36,00 & 18,00 & 18,00 & 11,00 & 18,00 & 13,00 & 20,00 \\
\hline $\begin{array}{c}\text { Tasa de } \\
\text { mortalidad (por } \\
\text { 1.000) }\end{array}$ & 12,00 & 7,00 & 7,00 & 11,00 & 6,00 & 8,00 & 8,00 \\
\hline $\begin{array}{c}\text { Esperanza de vida } \\
\text { al nacer }\end{array}$ & 58,00 & 77,00 & 70,00 & 76,00 & 74,00 & 78,00 & 70,00 \\
\hline $\begin{array}{c}\text { Tasa de } \\
\text { fecundidad global } \\
\text { (por mujer) }\end{array}$ & 4,70 & 2,50 & 2,20 & 1,60 & 2,20 & 1,90 & 2,50 \\
\hline $\begin{array}{c}\text { Tasa de } \\
\text { mortalidad } \\
\text { infantil (por 1.000 } \\
\text { nacidos vivos) }\end{array}$ & 74,00 & 24,00 & 39,00 & 6,00 & 19,00 & 6,00 & 44,00 \\
\hline $\begin{array}{c}\text { Tasa de migración } \\
\text { neta (por 1.000) }\end{array}$ & $-1,00$ & 5,00 & 0,04 & 2,00 & $-1,00$ & 3,00 & $\mathrm{n} / \mathrm{a}$ \\
\hline $\begin{array}{c}\text { Tasa de migración } \\
\text { interna 1990-2005 } \\
\text { (\%) }\end{array}$ & 15,40 & $\mathrm{n} / \mathrm{a}$ & $\mathrm{n} / \mathrm{a}$ & 22,30 & 19,30 & 17,80 & 17,50 \\
\hline $\begin{array}{c}\text { Población de 65 } \\
\text { años y más (\%) }\end{array}$ & 4,00 & 11,00 & 7,00 & 16,00 & 7,00 & 13,00 & 8,00 \\
\hline
\end{tabular}

Fuentes: LEVY; MOREL, 2012: 6, basada en PRB, 2011; UNDP, 2009. 
FIGURA 1 - Cambios en densidad población 1990-2005

Fuente: LEVY; MOREL, 2009: 9.

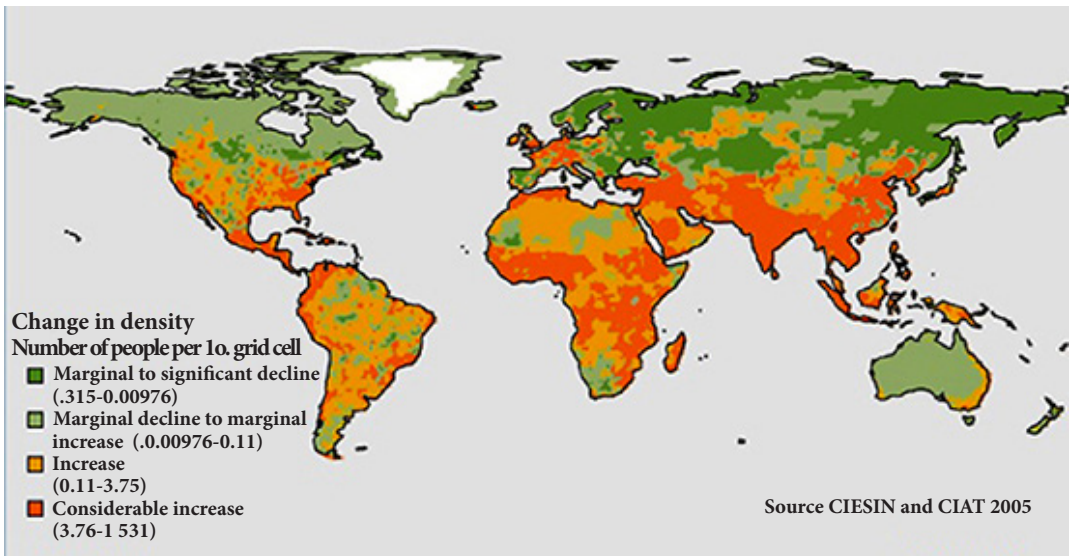

FIGURA 2 - Evolución de la población urbana y rural, América Latina - 1950-2050

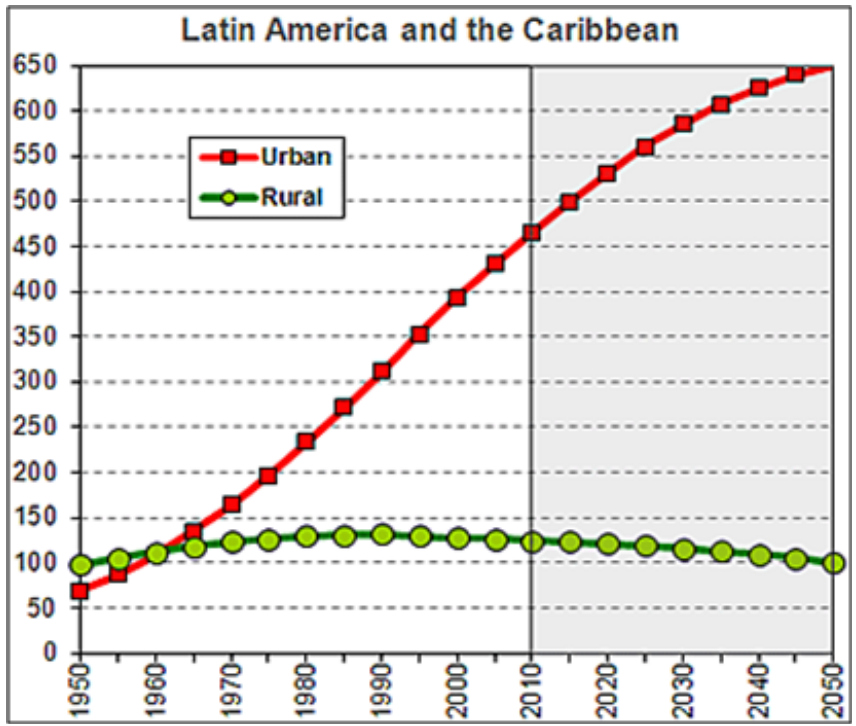

Fuente: UNITED NATIONS, 2011. 
Población y ambiente: cuestiones actuales...

Finalmente, se observa un renovado interés en todo lo relacionado con migración (en sentido amplio) y ambiente, en particular cambio climático (véase por ejemplo LACZKO; AGHAZARM, 2009; FORSIGHT, 2011; FUSSELL et al., 2014; OBOKATA et al., 2014). Algunas posibles razones para esto son las preocupaciones acerca de las consecuencias del cambio ambiental global para el bienestar humano y la movilidad de la población; el debate sobre la migración como estrategia de adaptación viable; la idea de que el desplazamiento por causas ambientales podría generar problemas de gobernanza y de seguridad; $y$, muy importante, nuevos resultados empíricos (ADAMO, 2010). El contexto de estas tendencias incluye la transformación de la geografía de la vulnerabilidad y el riesgo basado en el lugar, a través de rápida urbanización, poblaciones más móviles, globalización de los mercados y las comunicaciones, y creciente interconexión de personas y lugares (ADGER et al., 2009; SANDERSON, 2009).

\section{Referencias}

ADAMO, S. Environmental migration and cities in the context of global environmental change. Current Opinion in Environmental Sustainability, v. 2, p. 161-165, 2010. Disponível em: http://goo. gl/SdCUVq. Acesso em jul. 2015.

ADAMO, S. Reflexiones sobre la complejidad: investigación y formación en el área de estudios de población y medio ambiente. Ponencia presentada en el $1^{\text {o }}$ Taller sobre las Dimensiones Humanas del Cambio Ambiental en Argentina, Luján, Universidad Nacional de Luján, 8-10 agosto, 2007.

ADGER,W.N.;EAKIN, H.;WINKELS, A. Nested and teleconnected vulnerabilities to environmental change. Frontiers in Ecology and the Environment, v. 7, n. 3, p. 150-157, 2009. Disponível em: http://goo.gl/UqGZeR. Acesso em jul. 2015.

BLAIKIE, P.; CANNON, T.; DAVID, I.; WISNER, B. Vulnerabilidad 
el entorno social, político y económico de los desastres. $\mathrm{La}$ Red: Red de estudios sociales en prevención de desastres de América Latina, 1996. Disponível em: http://goo.gl/OwTdmS. Acesso em jul. 2015.

BOSERUP, E. [1965]. The conditions of agricultural growth: the economics of agrarian change under population pressure. New Brunswick (NJ), Aldine Transaction, 2005. Disponível em: https://goo.gl/yB83sf. Acesso em jul. 2015.

COMISSÃO ECONÔMICA PARA A AMÉRICA LATINA E O CARIBE (CEPAL). Efectos del cambio climático en la costa de América Latina y el Caribe: Impactos. Santiago de Chile, CEPAL, 2012. Disponível em: http://goo.gl/uN9zbJ. Acesso em jul. 2015.

DE SHERBININ, A.; CARR, D.; CASSELS, S.; JIANG, L. Population and environment. Annual Review of Environment and Resources, v. 32, p. 345-373, 2007. Disponível em: http://goo.gl/YpDjKQ. Acesso em jul. 2015.

FORESIGHT. Migration and Global Environmental Change. 2011. Future Challenges and Opportunities. Final project report. Executive Summary. London, 2011. Disponível em: https:// goo.gl/hwBqxM. Acesso em jul. 2015.

FUSSELL, E.; HUNTER, L. M.; GRAY, C. L. Measuring the environmental dimensions of human migration: The demographer's toolkit. Global Environmental Change, v. 28, p. 182-191, 2014. Disponível em: http://goo.gl/OSFV1m. Acesso em jul. 2015.

GLOBAL EXPERT PANEL. Demographic challenges for sustainable development. The Laxenburg declaration on Population and Sustainable Development. Options. Winter 2011/12. 2011. Disponível em: http://goo.gl/yUZVKO. Acesso em jul. 2015.

HUMMEL, D. et al. Inter-and transdisciplinary approaches to 
population-environment research for sustainability aims: a review and appraisal. Population and Environment, v. 34, n. 4, p. 481-509, 2013. Disponível em: http://goo.gl/9R4OeM. Acesso em jul. 2015.

HUNTER, L. M.; O'NEILL, B. C. Enhancing engagement between the population, environment, and climate research communities: the shared socio-economic pathway process. Population and environment, v. 35, n. 3, p. 231-242, 2014. Disponível em: http://goo.gl/y09NSK. Acesso em jul. 2015.

INTERNATIONAL SOCIAL SCIENCE COUNCIL (ISSC/ UNESCO). World social science report 2013: Changing global environments. Summary. Paris: OECD Publishing and UNESCO Publishing, 2013. Disponível em: http://goo.gl/ ZTNoch. Acesso em jul. 2015.

LACZKO, F.; AGHAZARM, C. (Eds.). Migration, environment and climate change: assessing the evidence. Geneva: OIM, 2009. Disponível em: http://goo.gl/9uiQPF. Acesso em jul. 2015.

LEVY, M.; MOREL, A. (coord. lead authors). Chapter 1: Drivers. In. Global environmental outlook GEO 5. UNEP, 2012. Disponível em: http://goo.gl/QQCpki. Acesso em jul. 2015.

LUTZ, W.; PRSKAWETZ, A.; SANDERSON, W. C. Introduction. Population and Environment Review, v. 28, p. 1-21, 2002.

MALTHUS, T. An Essay on the Principle of Population. 6th edition. London, John Murray, 1826. Disponível em: http://goo.gl/ kA6ELD. Acesso em jul. 2015.

MARANDOLA JR., E.; HOGAN, D. J. Vulnerabilidade do lugar vs. vulnerabilidade sociodemográfica: implicações metodológicas de uma velha questão. Revista Brasileira de Estudos de População, v. 26, n. 2, p. 161-181, 2009. Disponível em: http://goo.gl/BldqCh. Acesso em jul. 2015.

MARANDOLA JR., E; HOGAN, D. J. Em direção a uma demografia ambiental? Avaliação e tendências dos estudos de População 
e Ambiente no Brasil. Revista Brasileira de Estudos de População, v. 24, n. 2, p. 191-223, 2007. Disponível em: http://goo.gl/ WH0pIw. Acesso em jul. 2015.

MARQUETE, C. Population and environment relationships in developing countries: a select review of approaches and methods. Bergen, Chr. Michelsen Institute, 1997. Disponível em: http://goo.gl/sdrk1p. Acesso em jul. 2015.

MARTINE, G. et al. População e sustentabilidade na era das mudanças ambientais globais: contribuições para uma agenda brasileira. Belo Horizonte: ABEP, 2012.

OBOKATA, R.; VERONIS, L.; MCLEMAN, R. Empirical research on international environmental migration: a systematic review. Population and environment, v. 36, n. 1, p. 111-135, 2014. Disponível em: http://goo.gl/7eyYd2. Acesso em jul. 2015.

POPULATION REFERENCE BUREAU (PRB). World at 7 Billion: World Population Data Sheet 2011. Population Reference Bureau, Washington, DC, 2011. Disponível em: http://goo. gl/5RfwGb. Acesso em jul. 2015.

SANDERSON, M. R. Globalization and the environment: implications for human migration. Human Ecology Review, v. 16, n. 1, p. 93, 2009. Disponível em: http://goo.gl/k50IDb. Acesso em jul. 2015.

SCHENSUL, D.; DODMAN, D. Populating adaptation: incorporating population dynamics in climate change adaptation policy and practice. In. MARTINE, G.;SCHENSUL, D. (Eds). The Demography of Adaptation to Climate Change. New York, London and Mexico City: UNFPA, IIED, El Colegio de México, 2013. p. 1-23. Disponível em: http://goo.gl/a0zAWf. Acesso em jul. 2015.

UNITED NATIONS (UN). Department of Economic and Social Affairs. World Urbanization Prospects, the 2011 Revision. Disponível em: http://goo.gl/DgUSCI. Acesso em jul. 2015. 
Población y ambiente: cuestiones actuales...

UNITED NATIONS DEVELOPMENT PROGRAMME (UNDP). Human Development Report. United Nations Development Programme, New York, 2009. Disponível em: http://goo.gl/ UlJoVr. Acesso em jul. 2015.

VANWEY, L.; OSTROM, E.; MERETSKY, V. Theories underlying the study of human-environment interactions. En Moran, E. y E. Ostrom. Seeing the forest and the trees. Human-environment interactiosn in forest ecosystems. Cambridge, MA, MIT Press, 2005, p. 23-56.

ZABA, B.; CLARKE, J. Introduction: current directions in population-environment research. In. ZABA, B.; CLARKE, J. (Eds.). Environment and Population Change. Liege, Derouaux Ordina Editors, 1994, p. 9-43. 